\title{
Redox Regulation and Flower Development: A Novel Function for Glutaredoxins
}

\author{
S. Xing* , A. Lauri* , and S. Zachgo \\ Max Planck Institute for Plant Breeding Research, Carl-von-Linné-Weg 10, 50829 Köln, Germany \\ * These authors contributed equally to the work
}

Received: November 16, 2005; Accepted: May 2, 2006

\begin{abstract}
Glutaredoxins (GRXs) are small, ubiquitous oxidoreductases that have been intensively studied in $E$. coli, yeast and humans. They are involved in a large variety of cellular processes and exert a crucial function in the response to oxidative stress. GRXs can reduce disulfides by way of conserved cysteines, located in conserved active site motifs. As in E. coli, yeast, and humans, GRXs with active sites of the CPYC and CGFS type are also found in lower and higher plants, however, little has been known about their function. Surprisingly, 21 GRXs from Arabidopsis thaliana contain a novel, plant-specific CC type motif. Lately, information on the function of CC type GRXs and redox regulation, in general, is accumulating. This review focuses on recent findings indicating that GRXs, glutathione and redox regulation, in general, seem to be involved in different processes of development, so far, namely in the formation of the flower. Recent advances in EST and genome sequencing projects allowed searching for the presence of the three different types of the GRX subclasses in other evolutionary informative plant species. A comparison of the GRX subclass composition from Physcomitrella, Pinus, Oryza, Populus, and Arabidopsis is presented. This analysis revealed that only two CC type GRXs exist in the bryophyte Physcomitrella and that the CC type GRXs group expanded during the evolution of land plants. The existence of a large CC type subclass in angiosperms supports the assumption that their capability to modify target protein activity posttranslationally has been integrated into crucial plant specific processes involved in higher plant development.
\end{abstract}

Key words: Glutaredoxin, flower development, redox regulation, disulfide reduction.

\section{Glutaredoxins: Versatile Oxidoreductases Acting in Redox Regulation}

Glutaredoxins (GRXs) are oxidoreductases that belong to the thioredoxin (TRX) superfamily, all mediating reversible reduction of intracellular disulfide bonds in vivo (reviewed by Fernandes and Holmgren, 2004; Buchanan et al., 2005). The first

Plant Biol. 8 (2006): 547-555

(c) Georg Thieme Verlag KG Stuttgart · New York

DOI 10.1055/s-2006-924278 · Published online August 1, 2006

ISSN 1435-8603
GRX found was a dithiol hydrogen donor system for ribonucleotide reductase in an $E$. coli mutant lacking thioredoxin1 (Holmgren, 1976). Now, GRXs are known to exist in a number of isoforms in prokaryotes, viruses and eukaryotes, including yeast, vertebrates and humans, where they play a major role in the response to oxidative stress. Although several plant cDNAs have been isolated from spinach, poplar, Rhizinus and rice (Morell et al., 1995; Rouhier et al., 2003; Szederkényi et al., 1997; Lee et al., 2002), information on the function of plant GRXs has been scarce and analyses have mostly been of a descriptive nature. Recent reviews on plant glutaredoxins and plant redox control, in general, have comprehensively summarized the constantly accumulating knowledge within this field (Dietz, 2003; Lemaire, 2004; Rouhier et al., 2004; Buchanan et al., 2005; Ströher and Dietz, 2006). This review will survey novel findings indicating an intriguing function for plant GRXs and redox signalling in developmental processes. Furthermore, we have conducted a comparative analysis of the GRX organization in representative species from lower plants, gymnosperms and angiosperms, aiming for a better understanding of the evolution of the multiplicity of different GRX isoforms in photosynthetic organisms.

GRXs exert their activity through two different mechanisms that are depicted in Fig. 1. Both mechanisms depend on the presence of motifs containing characteristic cysteines with a sulfhydryl group that are particularly sensitive to reactive oxygen species (ROS) formed during oxidative stress (Gille and Sigler, 1995). In the dithiol reaction, two cysteines, normally arranged in an active site including a conserved C- and N-terminal cysteine $(\mathrm{CXXC})$, are required to reduce protein disulfides. The N-terminal cysteine initiates a nucleophilic attack on one of the sulfur atoms of the disulfide target. A transient disulfide bridge is formed between the two proteins which is resolved by generating an intramolecular disulfide bridge between the two cysteines of the active site, thereby releasing a reduced target protein (Fig. 1A). In the monothiol reaction, a protein tagged via a disulfide bridge with glutathione is reduced by means of only one, typically the $\mathrm{N}$-terminal, cysteine that can be part of either a CXXC or CXXS motif. Here, the GRX reacts exclusively with the glutathione (GSH), for which it possesses a high affinity (Fig. 1 B). Glutathionylation can protect sulfhydryl groups from irreversible damage during oxidative stress and, after normalization of the cellular redox state, protein reactivation occurs by deglutathionylation (Ghezzi, 2005). Monothiol reactions are only exerted by GRXs, whereas the di- 
A

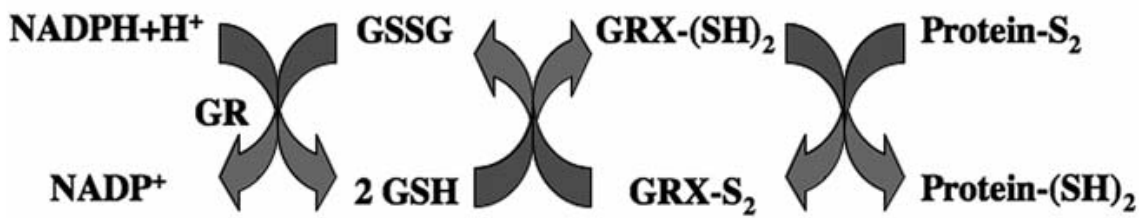

Fig. 1 GRX mechanisms for reduction of disulfides. (A) Dithiol mechanism: electrons are transferred from NADPH to glutathione reductase (GR), then to glutathione (GSH), and from there to glutaredoxin (GRX). Finally, GRX reduces disulfides in target proteins. (B) Monothiol mechanism: reduction of protein GSH mixed disulfides is called deglutathionylation (black arrows). Glutathionylation, the reverse reaction (grey arrows), generates glutathionylated proteins. (Adapted from Fernandes and Holmgren, 2004.)

B

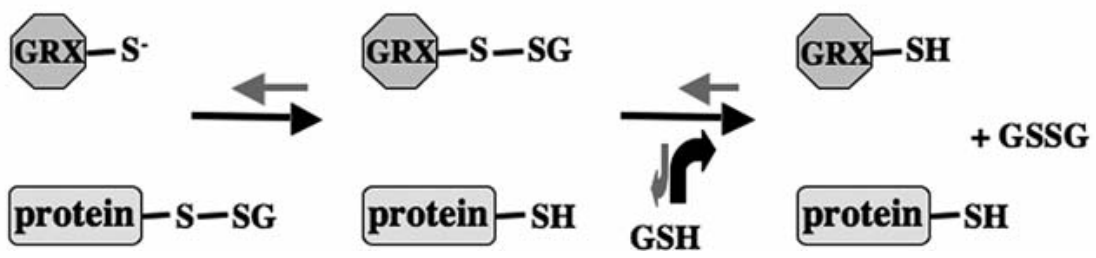

thiol mechanism can also be conducted by TRXs (Nulton-Persson et al., 2003). It is assumed that dithiol GRXs such as the CXXC types can exert dithiol and monothiol reductions, whereas monothiol CXXS type GRXs are restricted to reduction of mixed disulfides. Both disulfide reduction mechanisms have in common that they cause posttranslational modifications of GRX target proteins, probably in response to an altered cellular redox state. Consequently, protein structure can be altered, and these modifications represent a means by which the biological activity of target proteins can be regulated.

\section{The Family of Plant Glutaredoxins: Novel GRX Members Broaden the Horizon}

Based on the amino acid composition of the active motifs, the 31 members of the Arabidopsis GRXs family have been divided into three major subclasses (Lemaire, 2004; Rouhier et al., 2004). Six classical GRXs were identified in Arabidopsis and contain a CXXC motif, mostly represented by a CPYC active site (Lemaire, 2004). The monocysteinic enzymes of the CXXS type contain predominantly CGFS type active site motifs that were originally identified and characterized in yeast (RodríguezManzaneque et al., 1999). In Arabidopsis, this group comprises four GRXs. Surprisingly, the largest GRX group is also of the CXXC type, but these GRXs contain an additional, conserved cysteine in the second position of the motif that led to the designation of this novel CC type subclass (Lemaire, 2004). Database searches and protein sequence analysis from Lemaire (2004) and Rouhier et al. (2004) indicated that CC type GRXs might have appeared later during evolution and could be specific to higher plants. Intrigued by this observation, we took advantage of rapidly expanding plant EST and genome sequencing data and conducted a broader GRX search in selected plant species representing different stages of plant evolution. In databases from the cyanobacterium Synechocystis and green alga Chlamydomonas, we only identified GRXs of the CPYC and CGFS type but not of the CC type (data not shown), confirming earlier searches (Lemaire, 2004). As a representative for lower land plants, we searched the bryophyte model system Physco- mitrella patens EST and genomic sequence databases (the latter being a personal communication, S. Rensing, S. Zachgo). Next, we analyzed GRXs from seed plants and identified GRXs from the large collection of over 300000 ESTs from the gymnosperm Pinus taeda. The sequenced genome of Oryza sativa served as a representative for monocots and that of Populus, together with described GRXs from Arabidopsis (Rouhier et al., 2004; Lemaire, 2004), for dicots. All newly identified GRXs were aligned together using ClustalW. Fig. 2 gives the amino acid similarities, using the tree view program for graphic presentation. Clearly, extended analysis of GRX isoforms shows that they group into three subclasses, CPYC, CGFS and CC type, supporting data from earlier studies (Rouhier et al., 2004; Lemaire, 2004). The different GRXs from each species were listed according to their active site motif sequence and are shown in Fig. 3A. Strikingly, our data allow new insights into the evolution of the CC type GRX subclass. Two ESTs encoding CCMC type GRXs were identified in Physcomitrella. This bryophyte, thus far, represents the most basal plant species from which CC type GRXs could be identified. Searches were not successful in the green alga $C$. reinhardtii or in photosynthetically active cyanobacteria, however, with the limitation that these datasets do not yet represent all cDNAs. The occurrence of the CC type GRXs can, thus, probably be dated back about 450 million years, when the first nonvascular land plants evolved (Kenrick and Crane, 1997). In Fig. 3 B, a comparison of the number of members of the three GRX subclasses is depicted. This highlights another interesting observation: again, being aware of the limitation that not all GRXs might yet be annotated for all investigated species, it is still evident that the number of the CC type GRXs increases from bryophytes to gymnosperms and finally to angiosperms, represented by mono- and dicots. Numbers of CC type GRXs progressively increase from two for Physcomitrella to 22 and 21 CC type GRXs in Populus and Arabidopsis, respectively. In contrast, sizes of the other two GRX groups are similar, with 4-6 members of CPYC type GRXs and 3-8 members of CGFS type GRXs. Analysis of the different Arabidopsis GRXs, using expression profiling databases, revealed that most are expressed, supporting a 


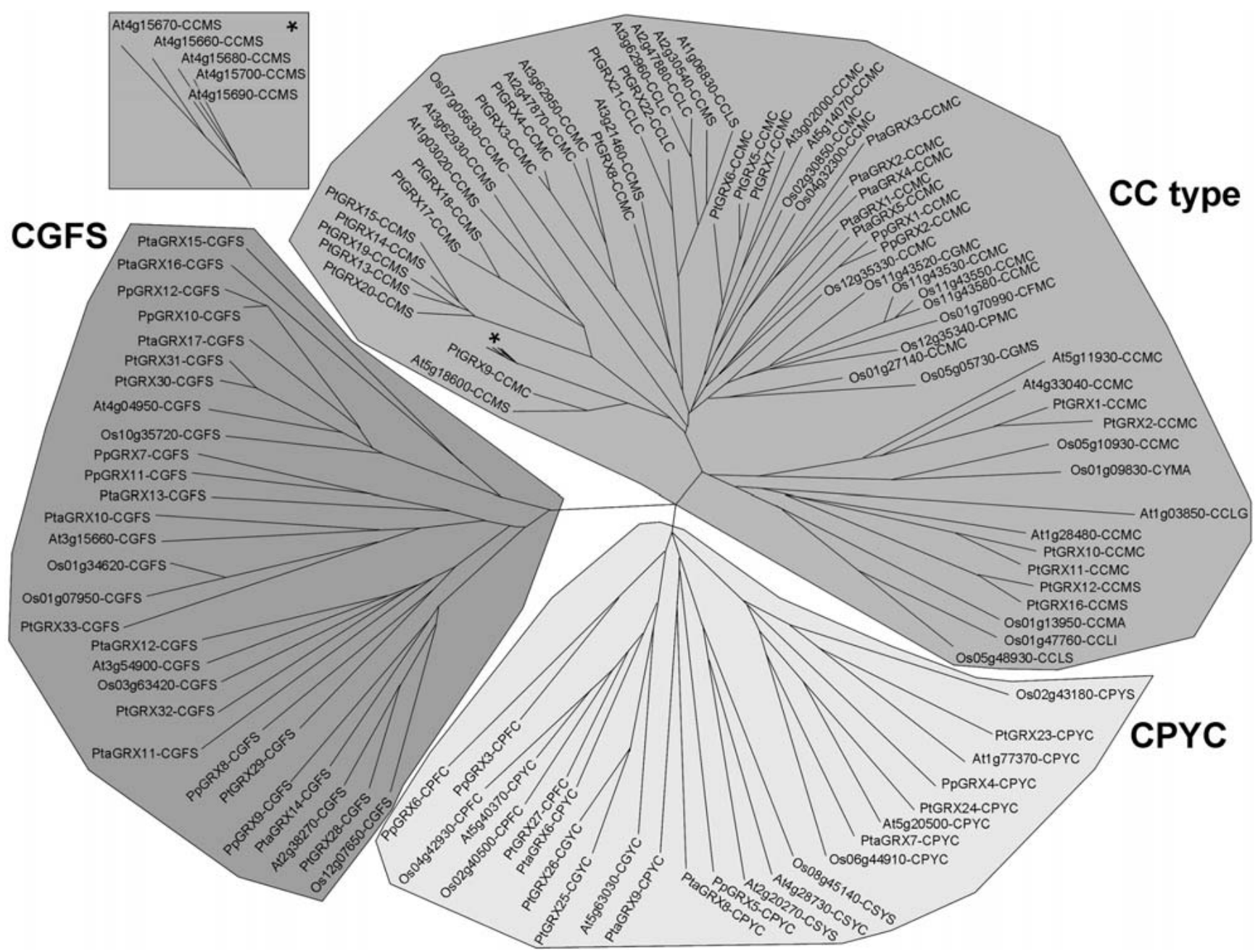

Fig. 2 Schematic representation of amino acid similarities between GRXs from Physcomitrella patens, Pinus taeda, Oryza sativa, Populus trichocarpa, and Arabidopsis thaliana. In order to retrieve the GRX amino acid sequences from Physcomitrella patens (Pp), Pinus taeda (Pta), and Oryza sativa (Os), the publicly available nucleotide sequences (containing ESTs and, for Pp, Os and Pt also genomic sequences) were searched using the tblastn program. Pp: http://moss.nibb.ac.jp, http://www. cosmoss.org; Pta: http://www.ncbi.nlm.nih.gov/Taxonomy/Browser/ wwwtax.cgi?id=3352; Os: http://www.tigr.org/tdb/e2k1/osa1/index. shtml; Pt: http://genome.jgi-psf.org/cgi-bin/runAlignment?db=Poptr1 \&advanced $=1$. The predicted amino acid sequences of ROXY1 (At3g02000), At4g04950 and At5g63030, representatives for the three different GRX subclasses from Arabidopsis, were used for the searches. Only nucleotide sequences from predicted proteins with an $E$ value $<10^{-5}$ were further grouped into contigs to determine the longest detectable unigene sequence. The corresponding translated amino acid

function for the CC type GRXs (data not shown). This observation raises the question of whether a relationship might exist between the development of more complex plant structures and the concomitant expansion of the CC type GRXs in land plants.

The CC type motif shows some features that distinguish this group from the other two subclasses. The function of the second conserved cysteine next to the $\mathrm{N}$-terminal cysteine remains elusive: is it also involved in disulfide reduction?; if so, via the sequence was submitted to the rpsblast program at the NCBI website in order to discriminate between GRX-encoding sequences and to exclude false positives, mainly originating from closely related TRXencoding sequences. The list of Arabidopsis GRXs was obtained from Rouhier et al. (2004). The predicted amino acid sequences from all the investigated organisms were aligned using the ClustalW program (http://www.ebi.ac.uk/clustalw) with the default settings. The alignment was then visualized with the treeview program (http://taxonomy.zoology.gla.ac.uk/rod/treeview.html). Amino acid sequences deduced from assembled contig sequences from Physcomitrella, Pinus, and Populus were numbered consecutively, following the order of those belonging to the CCMC, CPYC, and CGFS subclasses, and will be provided upon request. For identification of GRXs from Arabidopsis, the AGI code is given, and for GRXs from Oryza, locus identifiers from the TIGR rice genome annotation nomenclature are used.

monothiol or dithiol mechanism? However, this conserved cysteine was replaced in four GRXs from Oryza (Os01g70990 CFMC, Os11g43520 CGMC, Os05g05730 CGMS, Os01g09830 CYMA, Os12g35340 CPMC), although their overall amino acid similarity still groups these GRXs into the CC type subclass (Fig. 2).

The comparative study across different species allows further insights into deviations from the two active site motifs from the CPYC and CGFS subclasses (Fig. 3C). Proteins of the CPYC 
A

\begin{tabular}{|c|c|c|c|c|c|c|c|c|c|c|c|}
\hline \multicolumn{2}{|c|}{$\begin{array}{l}\text { Physcomitrella } \\
\text { patens }\end{array}$} & \multicolumn{2}{|c|}{ Pinus taeda } & Oryza sativa & \multicolumn{3}{|c|}{ Populus trichocarpa } & \multicolumn{4}{|c|}{ Arabidopsis thaliana } \\
\hline name & $\begin{array}{l}\text { active } \\
\text { site }\end{array}$ & ne & $\begin{array}{l}\text { active } \\
\text { site }\end{array}$ & $\begin{array}{lc}\text { name } & \begin{array}{c}\text { active } \\
\text { site }\end{array}\end{array}$ & ame & $\begin{array}{l}\text { active } \\
\text { site }\end{array}$ & $\begin{array}{cc}\text { name } & \begin{array}{c}\text { active } \\
\text { site }\end{array}\end{array}$ & name & $\begin{array}{l}\text { active } \\
\text { site }\end{array}$ & name & $\begin{array}{l}\text { active } \\
\text { site }\end{array}$ \\
\hline $\mathrm{X} 1$ & ССMC & PtaGRX1 & ССMC & Os02g30850 CCMC & PtGRX1 & ССMC & PtGRX18 CCMS & At1g28480 & ССMC & At5g18600 & CCMS \\
\hline & ССМС & & сСмС & $300 \mathrm{CCMC}$ & PtGRX2 & ССMC & X19 CCMS & 870 & & & \\
\hline $\mathrm{X} 3$ & CPEC & & $\rho \Omega$ & $9930 \mathrm{CC}$ & tGRX3 & ССMC & & & & & \\
\hline PpGRX4 & CPYC & $\mathrm{PtaC}$ & ССМС & 5630 ССMC & PtGRX4 & ССMC & $21 \mathrm{CCLC}$ & At3g62950 & ССMC & 850 & CCLG \\
\hline PpGRX5 & CPYC & PtaGRX5 & ССMC & $530 \mathrm{CCMC}$ & PtGRX5 & CCMC & 220 & 040 & ССMC & 830 & CCLS \\
\hline & CPFC & & CPY & $50 \mathrm{C}$ & & $\mathrm{CC}$ & $23 c$ & & & & \\
\hline & CGFS & & CPYC & $80 \mathrm{C}$ & & ССМС & & & & & \\
\hline PpGRX8 & CGFS & PtaGRX8 & CPYC & $330 \mathrm{CCMC}$ & PtGRX8 & ССMC & $25 \mathrm{C}$ & 20 & $\mathrm{CC}$ & 70 & CPYC \\
\hline PpGRX9 & CGFS & PtaGRX9 & CPYC & Os0 & PtGRX9 & ССMC & 260 & & & & CGYC \\
\hline & CGFS & & & $\mathrm{OC}$ & PtG & CCMC & Ptc & & & & \\
\hline $\mathrm{PpG}$ & CGFS & Pta & CGFS & $60 \mathrm{CCLI}$ & PtGR & ССMC & PtGRX28 CGFS & At3g62930 & CCMS & At2g20270 & CSYS \\
\hline \multirow[t]{5}{*}{ PpGRX12 } & CGFS & PtaGRX12 & CGFS & Os05g48930 CCLS & PtGRX12 & CCMS & PtGRX29 CGFS & At4g15660 & CCMS & At2g38270 & CGFS \\
\hline & & & & Os01g70990 CFMC & & & PtGRX30 CGFS & & CCMS & & CGFS \\
\hline & & & & $=0$ & $\mathrm{Pt}$ & & PtGRX31 C & AtA 1 & CCMS & At3 & COC \\
\hline & & & CGFS & Os05g05730 CGMS & PtG & & PtGRX32 CC & At4 & CCMS & At4g04950 & CGFS \\
\hline & & PtaGRX16 & CGFS & Os01g09830 CYMA & PtGRX16 & CCMS & PtGRX33 CGFS & At4g15700 & CCMS & & \\
\hline
\end{tabular}


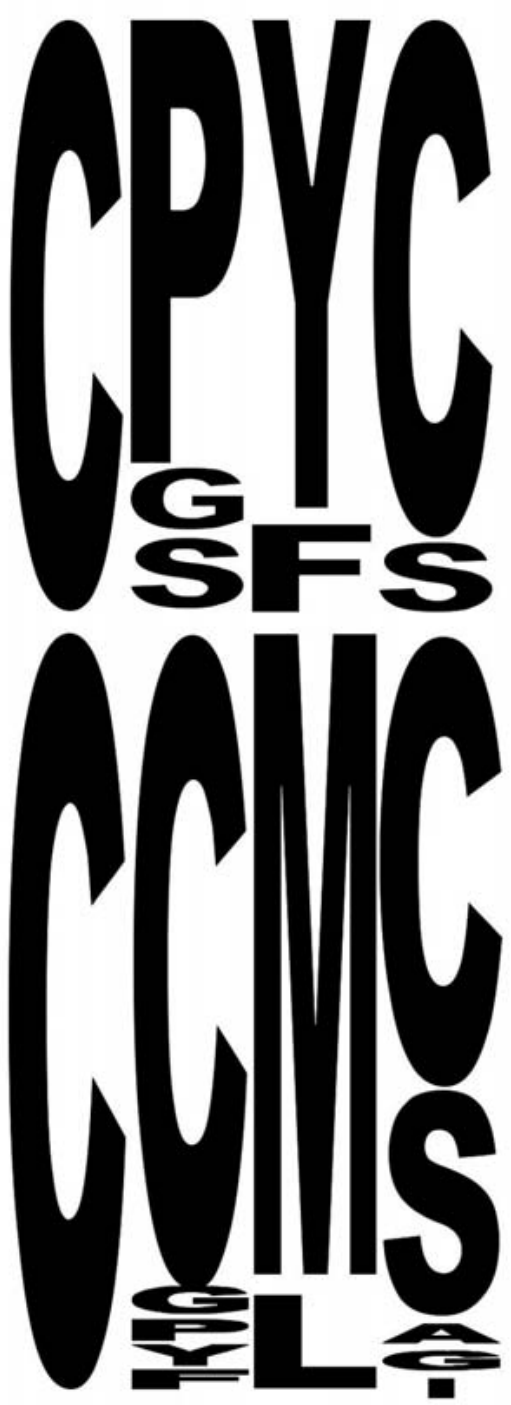

Fig. 3 
class were characterized initially from E. coli and S. cerevisiae. In plants, the most frequent sequence of their active site is CPYC. However, proline $(\mathrm{P})$ is replaced three times by a glycine $(\mathrm{G})$ and three times by a serine $(\mathrm{S})$ in 24 investigated CPYC motifs. The tyrosine $(\mathrm{Y})$ can be exchanged for a phenylalanine $(\mathrm{F})$, and the $\mathrm{C}$-terminal cysteine $(\mathrm{C})$ for a serine $(\mathrm{S})$. Members of the CGFS class are also present in many prokaryotic and eukaryotic organisms. These proteins show two peculiarities: firstly, the active site, CGFS, is absolutely conserved and we could not find a divergence from this motif composition, even in distantly-related species, such as Arabidopsis, Physcomitrella, and Agrobacterium tumefaciens (data not shown). Secondly, some members of this group, i.e., At4g04950 and PpGRX10, have a chimeric structure: two or three GRX domains at the C-terminus of the protein follow a TRX domain. This feature of CGFS type GRXs has also been demonstrated for proteins from many other organisms (Isakov et al., 2000).

\section{Function of Plant Glutaredoxins: Insights into a Black Box?}

Information on the biochemical activity, structure and function of plant glutaredoxins has thus far been limited to characterization of a few cDNAs, namely of the CPYC type, that were isolated from rice, spinach, Ricinus and poplar (Minakuchi et al., 1994; Szederkényi et al., 1997; Morell et al., 1995; Rouhier et al., 2001). Further analysis showed that some proteins possess activity in reduction assays using 2-hydroxylethyl disulfide (HED) and dehydroascorbate (DHA). The poplar GRX is a good electron donor for a cytosolic peroxiredoxin (Rouhier et al., 2001; Rouhier et al., 2002) and an Arabidopsis CC type GRX can regenerate a mitochondrial peroxiredoxin (Finkemeier et al., 2005). So far, the putative intracellular localization of GRXs has been determined using prediction software programs and, thereby, GRXs are expressed in all cellular compartments, except for the nucleus. However, localization is isoform-specific and different isoforms are restricted to different compartments (Rouhier et al., 2004). Evidence is accumulating that plant GRX proteins can be detected in the phloem sap in sieve tubes, indicating a function in long-distance signalling (Balachandran et al., 1997; Szederkényi et al., 1997; Rouhier et al., 2004).

However, recently, the first GRX mutant from Arabidopsis was isolated, allowing investigation of the function of a plant GRX in vivo. The gene was named ROXY1 and belongs to the CC type subclass. Three independent mutant alleles, all displaying the same phenotype, have been isolated from the GABI-KAT T-DNA collection (Xing et al., 2005). The roxy1 mutants show a floral

4 Fig. 3 Content of GRXs from five plant species. (A) GRXs identified from Physcomitrella patens, Pinus taeda, Oryza sativa, Populus trichocarpa, and GRXs reported from Arabidopsis thaliana were grouped together according to the amino acid composition of their active site. (B) Distribution of GRXs into different subclasses. For determining subclass membership, priority was given to overall amino acid similarity, even if amino acids in the name-giving motifs are not totally conserved. (C) Ratio composition of the active site sequence for CC type and CPYC proteins. Residue letter size is proportional to occurrence among the studied proteins. CPYC $(n=24)$ : first position $100 \%$ C; second position $75 \%$ P, 9\% G, $12.5 \%$ S, $12.5 \%$ G; third position $79 \%$ Y, $21 \%$ F; fourth position $87.5 \%$ C, $12.5 \%$ S. CC type $(n=67)$ : first position $100 \%$ C; second position $92.5 \% \mathrm{C}, 1.5 \% \mathrm{Y}, 1.5 \% \mathrm{~F}, 1.5 \% \mathrm{P}, 3.0 \% \mathrm{G}$; third position $88 \% \mathrm{M}$, $12 \% \mathrm{~L}$; fourth position $61 \% \mathrm{C}, 33 \% \mathrm{~S}, 3 \% \mathrm{~A}, 1.5 \% \mathrm{G}, 1.5 \% \mathrm{I}$. phenotype restricted to one floral organ. The number of roxy 1 petals is reduced from 4.0 to an average of 2.5. Petals that are formed also show defects during later morphogenesis and are often abnormally bent and form a reduced blade. ROXY1 mRNA expression was detected transiently in young floral buds and then in young floral organ primordia. Site-directed mutagenesis revealed that only the $\mathrm{N}$-terminal cysteine in the CCMC motif is crucial for ROXY1 function (Xing et al., 2005). This indicates that ROXY1 could exert its activity via the monothiol mechanism and posttranslationally modify target proteins by deglutathionylation. Further insight into ROXY1 function was obtained by double mutant analysis combined with expression studies. ROXY1 is required to restrict the expression of the MADS-box gene AGAMOUS $(A G)$, a key regulatory transcription factor controlling stamen and carpel organogenesis (Yanofsky et al., 1990) to the reproductive organs during wildtype flower development (Xing et al., 2005). Given the fact that $A G$ expression is controlled by a group of repressors (Conner and Liu, 2000; Franks et al., 2002), ROXY1 could exert its function by modifying the activity of $A G$ repressor proteins. Together, these results indicate an intriguing connection between redox regulation and control of floral organogenesis.

\section{Plant Glutaredoxin Targets}

Crucial to understanding the function of GRXs is the identification and analysis of the target proteins. Cellular redox homeostasis depends on the balance between oxidation and reduction of target thiols and dithiols, respectively. GRXs can either affect the glutathionylation status of target proteins via the monothiol mechanism or reduce intra- or intermolecular disulfide bridges via the dithiol mechanism.

In plants, the family of TRXs and their target proteins has been intensively studied and the number of TRX targets is rapidly expanding (Gelhaye et al., 2005; Buchanan and Balmer, 2005). Most TRX targets were identified using affinity chromatography approaches (Motohashi et al., 2001; Balmer et al., 2003; Lemaire et al., 2004). TRXs are stronger reductants than GRXs, with a midpoint redox potential of around $-300 \mathrm{mV}$, compared to $-200 \mathrm{mV}$ for GRXs (Åslund et al., 1997). Recently, Rouhier et al. (2005) identified targets for a plant GRX. Using liquid chromatography with a mutagenized second active site cysteine in the CPYC motif coupled to tandem mass spectrometry, over 90 targets of a polar GRX were isolated. Similar to observations for the TRX targets, the spectrum of these target proteins is very broad. The poplar GRX targets are involved in several processes, such as stress response, photorespiration, translation, nitrogen, sulfur and carbon metabolism and protein folding. Most of these proteins, being involved in housekeeping functions, are likely to be expressed at high levels and will therefore probably be detected with the techniques used by Rouhier et al. (2005). However, proteins involved in signalling or transcription factors that are normally expressed at a lower level might have escaped detection thus far. Using the tomato Cf-9 resistance protein in a yeast two-hybrid screen, a TRX-related protein (CITRX) from Lycopersicum esculentum was identified as an interaction partner. CITRX can alter protein kinase activity and is a negative regulator of cell death and defence mechanisms induced through the C-9 protein (Rivas et al., 2004). In the future, the challenges will be to isolate more targets and to test their specificity with complemen- 


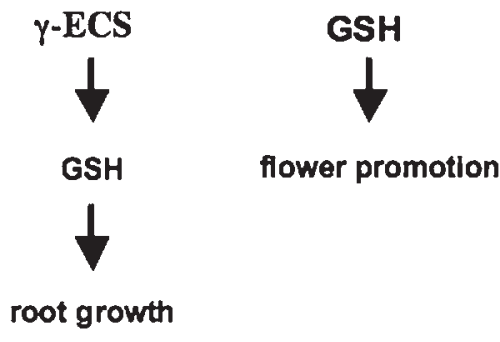

Fig. 4 Overview of redox-sensitive developmental processes. Different molecules, such as $\gamma$-ECS, GSH, ROXY1 (a CC type GRX), BOP1, and $B O P 2$ (transcriptional coactivators belonging to the NPR family), as well as the TGA transcription factor PAN, likely participate in the redox-sen-

tary approaches and thus determine if targets are modified by the dithiol or monothiol mechanism.

\section{Further Support for the Importance of Redox Regulation during Developmental Processes}

In yeast, the redox potential varies throughout the growth, division and apoptotic phases of its life cycle (Schafer and Buettner, 2001). Consequently, altered cellular redox states seem to correlate with altered developmental fates. In Fig. 4, a survey of currently known plant developmental processes affected by redox regulation is presented. This suggests that, besides participation in plant stress defence, redox-sensitive mechanisms seem to also participate in the control of flower and root development. In contrast to animals, plants use GSH as an electron donor for both the dithiol and monothiol reduction mechanisms (Fig. 1). This emphasizes the importance of GSH as a redox buffer that is present in plant cells at high levels of up to millimolar concentrations (Noctor and Foyer, 1998).

\section{Are similar signalling mechanisms involved in plant defence and floral development?}

Salicylic acid (SA) plays an important role in the onset of defence reactions against avirulent pathogens and mediates systemic acquired resistance (SAR) in plants (Delaney et al., 1994). SAR is a systemic and broad disease resistance characterized by the induction of pathogen-related (PR) genes exerting antimicrobial activity (Van Loon and Van Strien, 1999). NPR1 (NONEXPRESSOR OF PR GENES) is a positive regulator of the defence reaction in Arabidopsis (Dong, 2004). Upon SA accumulation, the intracellular redox potential is altered, intermolecular disulfide bonds holding NPR1 proteins together are reduced and thereby NPR 1 is converted into active monomers that are translocated from the cytoplasm into the nucleus (Mou et al., 2003). There, NPR1 acts as a coactivator of TGA transcription factors that recognize binding motifs in the $P R-1$ promoter and can activate PR-1 transcription. The NPR1/TGA interaction depends on the redox state of two cysteines in the TGA1 protein (Després et al., 2003). If an intramolecular disulfide bridge is formed between the two thiol groups in the TGA1 protein, interaction with NPR1 is abolished. NPR1 can only stimulate DNA binding activity of the reduced TGA1 protein. NPR1 con- tains a BTB/POZ domain and four ankyrin repeats mediating interaction with TGAs. These structural motifs are shared between five genes forming a small Arabidopsis gene family. Simultaneous inactivation of two other closely-related family members, BOP1 and BOP2, revealed a broad function for these NPR1-like genes in flower and leaf development (Ha et al., 2004; Hepworth et al., 2005; Norberg et al., 2005). Flowers of the double mutant form ectopic bracts and show organ abscission defects. Wild-type Arabidopsis flowers form four symmetrical sepals in the first whorl of the flower. In bop 1 bop2 flowers, an extra organ is formed in the first whorl and the two abaxial sepals are transformed into petaloid organs, thus giving rise to an asymmetric flower. Although most TGA genes are associated with the regulation of PR genes, it has been shown that one member of this bZIP transcription factor family, PERIANTHIA (PAN), is involved in the regulation of floral organ development (Chuang et al., 1999). In the pan mutant, an extra organ is formed in the first and second whorl in an abaxial position, disrupting flower symmetry and generating a similar phenotype to that found in bop1 bop2 flowers (Running and Meyerowitz, 1996). Biochemical interaction studies revealed that BOP1 and BOP2 proteins interact in yeast with PAN. As their expression patterns overlap in young flowers, this interaction is likely to occur in vivo. Further support for a function of BOP and PAN in the same genetic pathway was provided by analysis of the triple mutant (Hepworth et al., 2005). Given the similarities between the NPR1/TGA1 and BOP/PAN interaction it will be interesting to see if the BOP/PAN interaction might also be redox-controlled and thereby affects the activity of the PAN transcription factor during flower development.

\section{Flowering time and redox regulation: a connection?}

Glutathione (GSH) is a key component of plant antioxidant defence and is required for the dithiol and monothiol mechanism (Fig. 2). GSH is synthesized in two ATP-dependent steps, catalyzed by $\gamma$-glutamylcysteine synthase $(\gamma$-ECS) and glutathione synthase.

Recent data indicate that GSH plays a role in determining flowering time. Ogawa et al. (2004) investigated the relationship between GSH levels, photosynthesis and flowering in Arabi- 
dopsis and found that the level of GSH increases with light intensity. This requires $\gamma$-ECS catalyzing the key reaction of GSH synthesis. The level of GSH might be important in the regulation of flowering in Arabidopsis as it represents a pool of molecules that can be added posttranslationally to flowering timeassociated proteins and thereby influence their activity. Furthermore, mutants defective in glutathione biosynthesis or light harvesting antenna in photosystem II show reduced levels of GSH and have a late flowering phenotype (Ogawa et al., 2004). Thus, the level of GSH seems to be important in the regulation of flowering in Arabidopsis, which is further supported by the finding that a $\gamma$-ECS mutant (see below) also exhibits a late flowering phenotype (Cobbett et al., 1998).

\section{GSH participates in the regulation of cell division in the root apical meristem}

The rate-limiting step in synthesis of the tripeptide GSH is catalyzed by $\gamma$-ECS (May and Leaver, 1994). A mutation in the Arabidopsis $\gamma$-ECS (ROOT MERISTEMLESS1;RML1/CADMIUM SENSITIVE2) revealed that a glutathione-dependent pathway is required for correct cell division during postembryonic root development (Vernoux et al., 2000; Cobbett et al., 1998). Knockdown rml1 mutants are almost devoid of GSH and, consequently, cells in the root tip do not divide after germination, causing an arrest in root development at a length of about $1 \mathrm{~mm}$ (Vernoux et al., 2000). These data confirm earlier observations that GSH has a role in controlling cell division. High concentrations of GSH increased the number of cells undergoing mitosis, while depletion of GSH had the opposite effect (Sánchez-Fernández et al., 1997). The existence of a GSH-dependent transduction pathway has also been postulated for mammalian cells (Liang et al., 1989; Suthantrian et al., 1990). The $r m l 1$ phenotype is suggestive for the existence of a general GSH-dependent mechanism to regulate cell division and operates in a broad range of organisms.

\section{Conclusions and Outlook}

GRXs are oxidoreductases that can modify proteins posttranslationally and therefore have the potential to alter protein activity. They can reduce intra- and intermolecular disulfide bridges and also, in contrast to TRXs, reduce glutathionylated proteins and thus release deglutathionylated proteins. Evidence is accumulating for redox-regulated disulfide bridge formation, affecting DNA binding activity of plant transcription factors. Interestingly, these posttranslational modifications of TGA proteins are likely not only to be involved in signalling processes in stress responses, but also govern developmental processes. Glutathionylation of chaperones, kinases, metabolic enzymes and also cytoskeletal proteins occurs in bacteria, yeast and animal cells, especially in response to oxidative stress (Lind et al., 2002). Thus far, in Arabidopsis, two enzymes of sugar metabolism, a triose-phosphate isomerase and an aldolase, were shown to be glutathionylated and thus represent putative targets for GRXs (Ito et al., 2003). Furthermore, similar to disulfide bridge formation, glutathionylation represents a means by which the DNA binding activity of mammalian transcription factors can be altered, as shown for NF-kappaB and c-Jun (Pineda-Molina et al., 2001; Klatt et al., 1999). The evolution of regulatory transcription factors through gene duplication and subsequent functional divergence has been hypothesized to be a major driving force in the development of extant plants (Otto and Yong, 2002). In the future, it will be interesting to investigate if and how plant transcription factor activity is posttranslationally regulated, in addition to known modifications such as phosphorylation and prenylation, the latter also requiring a conserved cysteine (Fujita et al., 2003; Yalovsky et al., 2000).

The plant GRX family comprises three different subclasses: the classical CPYC and CGFS GRXs are present throughout prokaryotic and eukaryotic organisms, whereas the CC type GRXs have, thus far, only been identified from land plants. Our comparative analysis suggests that the expansion of this GRX subclass coincided with the evolution of more complex plants, increasing especially in seed plants, as compared to a more steady level in the other two subclasses. Analysis of mutants with defects at different levels of redox regulation supports the importance of this novel mechanism in the control of developmental processes, as outlined in Fig. 4. Genomic tools and recent progress in the field of plant proteomics will be extremely valuable in characterizing more GRXs and identifying and analyzing their targets in order to advance our understanding of how they might have contributed to the evolution of complex plant reproductive structures, namely the flower.

\section{Acknowledgements}

The authors thank Dr. Thomas Münster (MPIZ) and Mark Ziemann from our lab for helpful comments on the manuscript. We are grateful for support from Dr. Stefan Rensing (University of Freiburg) in the search for GRXs from Physcomitrella patens and for help with bioinformatic analyses from Dr. Heiko Schoof (MPIZ). S. Z. acknowledges support from the DFG. A. L. received a PhD fellowship from the DFG Graduierten Kolleg "Molecular analysis of developmental processes".

\section{References}

Åslund, F., Berndt, K. D., and Holmgren, A. (1997) Redox potentials of glutaredoxins and other thiol-disulfide oxidoreductases of the thioredoxin superfamily determined by direct protein-protein redox equilibria. Journal of Biological Chemistry 272, 30780 - 30786.

Balachandran, S., Xiang, Y., Schobert, C., Thompson, G. A., and Lucas, W. J. (1997) Phloem sap proteins from Cucurbita maxima and Ricinus communis have the capacity to traffic cell to cell through plasmodesmata. Proceedings of the National Academy of Sciences of the USA 94, 14150- 14155.

Balmer, Y., Koller, A., del Val, G., Manieri, W., Schürmann, P., and Buchanan, B. B. (2003) Proteomics gives insight into the regulatory function of chloroplast thioredoxins. Proceedings of the National Academy of Sciences of the USA 100, 370-375.

Buchanan, B. B. and Balmer, Y. (2005) REDOX REGULATION: a broadening horizon. Annual Review of Plant Biology 56, 187-220.

Chuang, C. F., Running, M. P., Williams, R. W., and Meyerowitz, E. M. (1999) The PERIANTHIA gene encodes a bZIP protein involved in the determination of floral organ number in Arabidopsis thaliana. Genes and Development 3, 334-344.

Cobbett, C. S., May, M. J., Howden, R., and Rolls, B. (1998) The glutathione-deficient, cadmium-sensitive mutant, cad2-1, of Arabidopsis thaliana is deficient in gamma-glutamylcysteine synthetase. The Plant Journal 16, $73-78$.

Conner, J. and Liu, Z. (2000) LEUNIG, a putative transcriptional corepressor that regulates AGAMOUS expression during flower development. Proceedings of the National Academy of Sciences of the USA 97, 12902 - 12907. 
Delaney, T. P., Uknes, S., Vernooij, B., Friedrich, L., Weymann, K., Negrotto, D., Gaffney, T., Gutrella, M., Kessmann, H., Ward, E., and Ryals, J. (1994) A central role of salicalic acid in plant disease resistance. Science 266, $1247-1250$.

Després, C., Chubak, C., Rochon, A., Clark, R., Bethune, T., Desveaux, D., and Fobert, P. R. (2003) The Arabidopsis NPR1 disease resistance protein is a novel cofactor that confers redox regulation of DNA binding activity to the basic domain/leucine zipper transcription factor TGA1. Plant Cell 15, 2181 - 2191.

Dietz, K. J. (2003) Redox control, redox signaling, and redox homeostasis in plant cells. International Review of Cytology 228, $141-$ 193.

Dong, X. (2004) NPR1, all things considered. Current Opinion in Plant Biology 7, 547-552.

Fernandes, A. P. and Holmgren, A. (2004) Glutaredoxins: glutathione-dependent redox enzymes with functions far beyond a simple thioredoxin backup system. Antioxidants and Redox Signaling 6, 63-74.

Finkemeier, I., Goodman, M., Lamkemeyer, P., Kandlbinder, A., Sweetlove, L. J., and Dietz, K. J. (2005) The mitochondrial type II peroxiredoxin $\mathrm{F}$ is essential for redox homeostasis and root growth of Arabidopsis thaliana under stress. Journal of Biological Chemistry $280,12168-12180$.

Franks, R. G., Wang, C., Levin, J. Z., and Liu, Z. (2002) SEUSS, a member of a novel family of plant regulatory proteins, represses floral homeotic gene expression with LEUNIG. Development 129, 253 - 263.

Fujita, H., Takemura, M., Tani, E., Nemoto, K., Yokota, A., and Kohchi, T. (2003) An Arabidopsis MADS-box protein, AGL24, is specifically bound to and phosphorylated by meristematic receptor-like kinase (MRLK). Plant Cell Physiology 44, 735 - 742.

Ghezzi, P. (2005) Regulation of protein function by glutathionylation. Free Radical Research 39, 573-580.

Gelhaye, E., Rouhier, N., Navrot, N., and Jacquot J. P. (2005) The plant thioredoxin system. Cellular and Molecular Life Sciences 62, $24-$ 35.

Gille G. and Sigler K. (1995) Oxidative stress and living cells. Folia Microbiol 40, 131 - 152.

Ha, C. M., Jun, J. H., Nam, H. G., and Fletcher, J. C. (2004) BLADE-ONPETIOLE1 encodes a BTB/POZ domain protein required for leaf morphogenesis in Arabidopsis thaliana. Plant Cell Physiology 45, $1361-1370$

Hepworth, S. R., Zhang, Y., McKim, S., Li, X., and Haughn, G. W. (2005) BLADE-ON-PETIOLE-dependent signaling controls leaf and floral patterning in Arabidopsis. Plant Cell 17, 1434-1448.

Holmgren, A. (1976) Hydrogen donor system for Escherichia coli ribonucleoside-diphosphate reductase dependent upon glutathione. Proceedings of the National Academy of Sciences of the USA 73, $2275-2779$.

Isakov, N., Witte, S., and Altman, A. (2000) PICOT-HD: a highly conserved protein domain that is often associated with thioredoxin and glutaredoxin modules. Trends Biochemical Science 25, 537 539.

Ito, H., Iwabuchi, M., and Ogawa, K. (2003) The sugar-metabolic enzymes aldolase and triose-phosphate isomerase are targets of glutathionylation in Arabidopsis thaliana: detection using biotinylated glutathione. Plant Cell and Physiology 44, 655-660.

Kenrick, P. and Crane, P. R. (1997) The origin and early evolution of plants on land. Nature 389, $33-38$.

Klatt, P., Molina, E. P., de Lacoba, M. G., Padilla, C. A., Martinez-Galesteo, E., Barcena, J. A., and Lamas, S. (1999) Redox regulation of c-Jun DNA binding by reversible S-glutathionylation. FASEB Journal 13,1481-1490.

Lee, K. O., Lee, J. R., Yoo, J. Y., Jang, H. H., Moon, J. C., Jung, B. G., Chi, Y. H., Park, S. K., Lee, S. S., Lim, C. O., Yun, D. J., Cho, M. J., and Lee, S. Y. (2002) GSH-dependent peroxidase activity of the rice (Oryza sativa) glutaredoxin, a thioltransferase. Biochemical and Biophysical Research Communications 296, 1152 - 1156.
Lemaire, S. D. (2004) The glutaredoxin family in oxygenic photosynthetic organisms. Photosynthesis Research 79, 305-318.

Liang, C. M., Lee, N., Cattell, D., and Liang, S. M. (1989) Glutathione regulates interleukin-2 activity on cytotoxic T-cells. Journal of Biological Chemistry 264, 13519-13523.

Lind, C., Gerdes, R., Hamnell, Y., Schuppe-Koistinen, I., Brockenhuus von Löwenhielm, H., Holmgren, A., and Cotgreave, I. A. (2002) Identification of S-glutathionylated cellular proteins during oxidative stress and constitutive metabolism by affinity purification and proteomic analysis. Archives of Biochemistry and Biophysics 406, $229-240$.

May, M. J. and Leaver, C. J. (1994) Arabidopsis thaliana gamma-glutamylcysteine synthetase is structurally unrelated to mammalian, yeast, and Escherichia coli homologs. Proceedings of the National Academy of Sciences of the USA 91, 10059-10063.

Minakuchi, K., Yabushita, T., Masumura, T., Ichihara, K., and Tanaka, K. (1994) Cloning and sequence analysis of a cDNA encoding rice glutaredoxin. FEBS Letters 337, 157-160.

Morell, S., Follmann, H., and Haberlein, I. (1995) Identification and localization of the first glutaredoxin in leaves of a higher plant. FEBS Letters 369, 149-152.

Motohashi, K., Kondoh, A., Stumpp, M. T., and Hisabori, T. (2001) Comprehensive survey of proteins targeted by chloroplast thioredoxin. Proceedings of the National Academy of Sciences of the USA 98, 11224- 11229 .

Mou, Z., Fan, W., and Dong, X. (2003) Inducers of plant systemic acquired resistance regulate NPR1 function through redox changes. Cell 113, $935-944$.

Noctor, G. and Foyer, C. H. (1998) ASCORBATE and GLUTATHIONE: keeping active oxygen under control. Annual Review of Plant Physiology and Plant Molecular Biology 49, 249-279.

Norberg, M., Holmlund, M., and Nilsson, O. (2005) The BLADE ON PETIOLE genes act redundantly to control the growth and development of lateral organs. Development 132, 2203-2213.

Nulton-Persson, A. C., Starke, D. W., Mieyal, J. J., and Szweda, L. I. (2003) Reversible inactivation of alpha-ketoglutarate dehydrogenase in response to alterations in the mitochondrial glutathione status. Biochemistry 42, 4235-4242.

Ogawa, K., Hatano-Iwasaki, A., Yanagida, M., and Iwabuchi, M. (2004) Level of glutathione is regulated by ATP-dependent ligation of glutamate and cysteine through photosynthesis in Arabidopsis thaliana: mechanism of strong interaction of light intensity with flowering. Plant Cell and Physiology 45, 1-8.

Otto, S. P. and Yong, P. (2002) The evolution of gene duplicates. Advances in Genetics 46, $451-483$.

Pineda-Molina, E., Klatt, P., Vazquez, J., Marina, A., Garcia de Lacoba, M., Perez-Sala, D., and Lamas, S. (2001) Glutathionylation of the p50 subunit of NF-kappaB: a mechanism for redox-induced inhibition of DNA binding. Biochemistry 40, 14134-14142.

Rivas, S., Rougon-Cardoso, A., Smoker, M., Schauser, L., Yoshioka, H., and Jones, J. D. G. (2004) CITRX thioredoxin interacts with the tomato Cf- 9 resistance protein and negatively regulates defence. EMBO Journal 23, 2156-2165.

Rodríguez-Manzaneque, M. T., Ros, J., Cabiscol, E., Sorribas, A., and Herrero, E. (1999) Grx5 glutaredoxin plays a central role in protection against protein oxidative damage in Saccharomyces cerevisiae. Molecular and Cellular Biology 19, 8180-8190.

Rouhier, N., Gelhaye, E., Sautiere, P. E., Brun, A., Laurent, P., Tagu, D., Gerard, J., de Faÿ, E., Meyer, Y., and Jacquot, J. P. (2001) Isolation and characterization of a new peroxiredoxin from poplar sieve tubes that uses either glutaredoxin or thioredoxin as a donor. Plant Physiology 127, 1299-1309.

Rouhier, N., Gelhaye, E., and Jacquot J. P. (2002) Glutaredoxin-dependent peroxiredoxin from poplar: protein-protein interaction and catalytic mechanism. Journal of Biological Chemistry 277, 1360913614. 
Rouhier N., Vlamis-Gardikas, A., Lillig, C. H., Berndt, C., Schwenn, J. D., Holmgren, A., and Jacquot, J. P. (2003) Characterization of the redox properties of poplar glutaredoxin. Antioxidants and Redox Signaling 5, 15-22.

Rouhier, N., Gelhaye, E., and Jacquot, J. P. (2004) Plant glutaredoxins: still mysterious reducing systems. Cellular and Molecular Life Sciences $61,1266-1277$.

Rouhier, N., Villarejo, A., Srivastava, M., Gelhaye, E., Keech, O., Droux, M., Finkemeier, I., Samuelsson, G., Dietz, K. J., Jacquot, J. P., and Wingsle, G. (2005) Identification of plant glutaredoxin targets. Antioxidants and Redox Signaling 7, 919-929.

Running, M. P. and Meyerowitz, E. M. (1996) Mutations in the PERIANTHIA gene of Arabidopsis specifically alter floral organ number and initiation pattern. Development 122, 1261 - 1269.

Sánchez-Fernández, R., Fricker, M., Corben, L. B., White, N. S., Sheard, N., Leaver, C. J., Van Montagu, M., Inzé, D., and May, M. J. (1997) Cell proliferation and hair tip growth in the Arabidopsis root are under mechanistically different forms of redox control. Proceedings of the National Academy of Sciences of the USA 94, 2745-2750.

Schafer, F. Q. and Buettner G. R. (2001) Redox environment of the cell as viewed through the redox state of the glutathione disulfide/ glutathione couple. Free Radical Biology and Medicine 30, 1191 1212.

Ströher, E. and Dietz, K. J. (2006) Concepts and approaches towards understanding the cellular redox proteome. Plant Biology 8, 407418.

Suthantrian, M., Anderson, M. E., Sharma, V. K., and Meister, A. (1990) Glutathione regulates activation-dependent DNA synthesis in highly purified normal human $\mathrm{T}$ lymphocytes via the CD2 and CD3 antigens. Proceedings of the National Academy of Sciences of the USA 87, $3343-3347$.

Szederkényi, J., Komor, E., and Schobert, C. (1997) Cloning of the cDNA for glutaredoxin, an abundant sieve-tube exudate protein from Ricinus communis L. and characterisation of the glutathionedependent thiol-reduction system in sieve tubes. Planta 202, $349-356$.

Van Loon, L. C. and Van Strien, E. A. (1999) The families of pathogenesis-related proteins, their activities, and comparative analysis of PR-1 type proteins. Physiological and Molecular Plant Pathology $55,85-97$.

Vernoux, T., Wilson, R. C., Seeley, K. A., Reichheld, J. P., Muroy, S., Brown, S., Spencer C., Maughan, S. C., Cobbett, C. S., Van Montagu, M., Inzé, D., May, M. J., and Sung, Z. R. (2000) The ROOT MERISTEMLESS1/CADMIUM SENSITIVE2 gene defines a glutathione-dependent pathway involved in initiation and maintenance of cell division during postembryonic root development. Plant Cell 12, $97-$ 110.

Xing, S., Rosso, M. G., and Zachgo, S. (2005) ROXY1, a member of the plant glutaredoxin family, is required for petal development in Arabidopsis thaliana. Development 132, 1555-1565.

Yanofsky, M. F., Ma, H., Bowman, J. I., Drews, G. N., Feldmann, K. A., and Meyerowitz, E. M. (1990) The protein encoded by the Arabidopsis homeotic gene agamous resembles transcription factors. Nature 346, 35-39.

Yalovsky, S., Rodriguez-Concepcion, M., Bracha, K., Toledo-Ortiz, G., and Gruissem, W. (2000) Prenylation of the floral transcription factor APETALA1 modulates its function. Plant Cell 12,1257-1266.

\section{S. Zachgo}

Max Planck Institute for Plant Breeding Research Carl-von-Linné-Weg 10 50829 Köln

Germany

E-mail: szachgo@mpiz-koeln.mpg.de

Editor: B. Schulz 